\title{
A Model for Nonlinear Dynamic Systems with Application Potential in Management
}

\author{
Mihai PASCADI \\ University "Politehnica" of Bucharest, Romania \\ mihai.pascadi@avantera.ro
}

\begin{abstract}
Systems' modeling represents a valuable management instrument. The paper proposes a possible modeling method for the behavior of economic (or other type of) non-linear dynamic systems.

To this end, it uses a particular (input-state-output) vector sample based function to approximate the n-dimensional vector field describing the modeled system.

The paper presents the approximation algorithm that was developed and further tested with good results on a limited (yet) set of data.

The approach enables the development of future trainable models, convergent to the "to be modeled" systems, task considered the next step to be done.

The main benefit resides in the possibility to develop models in order to simulate and predict evolutions, may it be the evolution of a product in a market, of an institution/company in its environment or of another economic sub-system of interest.
\end{abstract}

Keywords: model, non-linear dynamic systems, systems management

\section{INTRODUCTION}

Few if any of the large and complex economic systems are indeed predictable (KeilisBorok, 2011). In many cases, predictions are based on qualitative considerations. Insights regarding the "actual" model of a system and the way it works are frequently empirical.

The huge interest for the subject (around 2450000 hits on Google for "conferences on economic modeling") (***, Wikipedia) should be enough to justify the effort of developing a model and an algorithm that should converge to the approximated system based on accumulation of information regarding the states the system proves to find itself in its evolution. Each such state becomes information to be used in adapting / training the approximating model to better reflect the behavior of the approximated system. 


\section{Hypotheses}

H1. The approximated system is describable by a continuous function. Even if not true in many cases, the approximation through an analytical function that is continuous is beneficial. The impact of this hypothesis has not yet been evaluated but it is part of the further research plan.

H2. Any kind of model built is supposed to be fully described (a full set of input values, parameters and output values is available). Of course, in the absence of a part of the necessary data the model could either decrease its precision in making predictions or could even become unable make any predictions.

\section{Limits of the model}

The theoretical limits of this model are the following:

L1. The approximated function is "known" only through a limited number of values corresponding to a finite number of points ( $\mathrm{T}$ points). There is infinity of functions that pass exactly through those points. When developing the approximating function, only the smoother functions will be well approximated.

L2. Having only information regarding $\mathrm{T}$ points, the convergence can be discussed only in relationship with those points and not with a given continuous function.

L3. Determining the trajectory of the non-linear dynamic system is as well subject to approximations, on top of limits discussed above.

\section{APPROXIMATING THE NONLINEAR SYSTEM FUNCTION}

We consider a general form for a dynamic system $(* * *$, Wikipedia):

$$
\dot{x}=\boldsymbol{F}(\boldsymbol{x})
$$

where $\mathrm{x}, \mathrm{F}(\mathrm{x}) \in \mathbf{R}^{\mathrm{n}}$. The non-linearity of the above system supposes that

$$
F(a * x+b * y)<>a F(x)+b F(y)
$$

but the proposed method would be applicable both to non-linear and linear systems (for which a much richer range of methods exists).

Comment: the chosen representation of the dynamic system covers the entire relationship between inputs, state parameters and outputs of the system (which would be the traditional representation of a system, as in Figure 1).

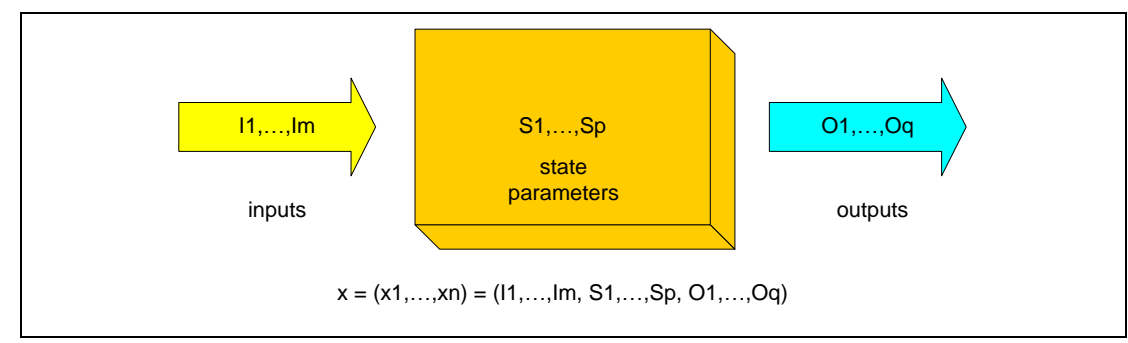

Figure 1: Dynamic system input-state-output representation 
Once the function $\mathrm{F}$ is known, the evolution of the system itself could be determined with any of the known integration (numerical) methods (Atkinson, 2009). A transformation of (1) into a discrete dynamical system leads to a difference equation:

$$
\boldsymbol{x}_{\boldsymbol{i}+\mathbf{1}}-\boldsymbol{x}_{\boldsymbol{i}}=\boldsymbol{F}\left(\boldsymbol{x}_{\boldsymbol{i}}\right) *\left(\boldsymbol{t}_{\boldsymbol{i}+\mathbf{1}}-\boldsymbol{t}_{\boldsymbol{i}}\right)
$$

which could serve as well in determining approximated future states of the system.

Thus, the first step in determining the future evolution of a system is to determine its function $\mathrm{F}(\mathrm{x})$.

\section{The approximating function}

Let's consider that the observation of the approximated system has led to the following set of data:

$x_{1}, \ldots, x_{T}$, where, $x_{t} \in \mathbf{R}^{n}-$ called the approximation points

and $x_{t+1}=F\left(x_{t}\right) *\left(t_{t+1}-t_{t}\right)$

with $F=\left(f_{1}, \ldots, f_{n}\right)$,

that represent successive states of "to be" approximated system.

The task is to build an approximating function for $\mathrm{F}$ based on this data. A wide variety of methods exist already (Judd, 2012). In order to choose the right solution (existing or new method), the following design specifications have been drawn (S1 ... S4).

S1. The approximating function should converge to the "to be approximated" - called from now on "approximated" function in the above T points of $\mathbf{R}^{n}$;

$\mathrm{S} 2$. For each component $f_{i}$ of $\mathrm{F}$, the approximating function should be built as a sum of simple functions $\varphi_{t}$ that each of them have relevant value in the vicinity of one of the approximation points and are tending to zero the farther we are from that vicinity; in this case, we will have $\mathbf{T}$ simple functions, one for each approximation point and for each component $f_{i}$ of $\mathrm{F}$;

S3. As the approximated function is supposed to be continuous, we should choose a continuous approximating function;

S4. The $\mathbf{T}$ approximating simple functions $\varphi_{t}$ for each component $f_{i}$ of $\mathrm{F}$ should have more than one parameter in their expression, allowing in this case the imposition of additional conditions.

Based on the above considerations (especially S2), a new method has been developed and the chosen expression for the scalar simple functions $\varphi_{t}$ is:

where

$$
\varphi_{t} \stackrel{\text { def }}{=} \frac{a_{t}}{\left(b_{t} *\left(x-x_{t}\right)^{2}+1\right)}
$$

$a_{t}, b_{t}$ are parameters while $x_{t}$ is one of $T$ approximating points in $\boldsymbol{R}^{n}$ and $\left(x-x_{t}\right)^{2}$ is the scalar product of the difference vector $x-x_{t}$ with itself, $x_{t}$ is an approximation point and $\mathrm{x}$ is another point in $\mathbf{R}^{n}$ where the approximated value of $\mathrm{F}$ is to be calculated. 
The chosen function meets the above design specifications. The component $f_{i}$ of $\mathrm{F}$ will have thus the form:

$f_{i}=\sum_{t=1}^{T} \varphi_{t}$, with $f_{i}, \varphi_{t} \in \mathbf{R}$.

Since the approximation of the vector function $\mathrm{F}$ is reduced to the approximation of its components, further, we shall discuss the approximation of the scalar function $f_{i}$.

\section{The approximation algorithm}

Corresponding to each component $f_{i}$ of $\mathrm{F}$, we extract from the given set of data the relevant components that specify for each $x_{t} \in \mathbf{R}^{n}$ the $y_{t}$ values $f_{i}$ should have for each approximating point $\left(f_{i}\left(x_{t}\right)=x_{t+1}^{i} \stackrel{\text { def }}{=} y_{t}\right)$.

The first iteration of the approximating function is defined as follows:

1. Determine the minimum and maximum values of $y_{t}, y_{m n}$ and $y_{m x}$ and their corresponding approximating points $x_{m n}$ and $x_{m x}$.

2. Determine the parameters for the first iteration of the approximating function, corresponding to $x_{m n}$ and $x_{m x}$ :

$$
\begin{aligned}
f_{i}^{1}(x)=\varphi_{m n}+ & \varphi_{m x} \\
& =\frac{a_{m n}}{\left(b_{m n} *\left(x-x_{m n}\right)^{2}+1\right)}+\frac{a_{m x}}{\left(b_{m x} *\left(x-x_{m x}\right)^{2}+1\right)}
\end{aligned}
$$

by imposing the following conditions:

$$
\begin{gathered}
f_{i}^{1}\left(x_{m n}\right)=y_{m n} \\
f_{i}^{1}\left(x_{m x}\right)=y_{m x} \\
f_{i}^{1}\left(\frac{x_{m n}+x_{m x}}{2}\right)=\frac{y_{m n}+y_{m x}}{2}
\end{gathered}
$$

3. Determine the next-iteration-to-approximate-values :

$$
y_{t}^{2}=y_{t}-f_{i}^{1}\left(x_{t}\right)
$$

by substracting the approximated through $f_{i}$ values of $y_{t}$ from the actual values of $y_{t}$. For a certain iteration " $k$ ", the steps would be similar (Figure 2):

1. Determine the minimum and maximum values of $y_{t}^{k}, y_{m n}^{k}$ and $y_{m x}^{k}$, and their corresponding approximating points $x_{m n}^{k}$ and $x_{m x}^{k}$.

2. Determine the parameters for the k-th iteration of the approximating function, corresponding to $x_{m n}^{k}$ and $x_{m x}^{k}$

3. Decide if the stop criterion is met or determine the $(\mathrm{k}+1)$-iteration-toapproximate-values. 


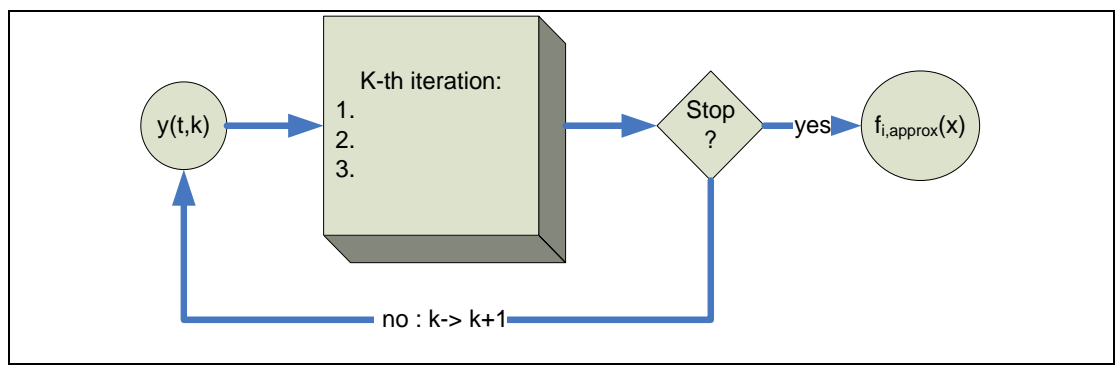

Figure 2: Approximation algorithm representation

The following questions need answer:

- How to calculate the parameters $a_{m n}, a_{m x}, b_{m n}, b_{m x}$ ?

- Which could be the Stop criterion?

The parameters' expressions are determined by conditions (7), (8) and (9). In addition, as we have three conditions and 4 parameters, we may add the supplementary condition

The results are the following:

$$
b_{m n}=b_{m x}=b \text {. }
$$

$$
\begin{aligned}
a_{m n} & =\frac{9}{8} *\left(y_{m n}+\frac{y_{m x}}{3}\right) \\
a_{m x} & =\frac{9}{8} *\left(y_{m x}+\frac{y_{m n}}{3}\right) \\
b & =\frac{2}{\left(x_{m x}-x_{m n}\right)^{2}}
\end{aligned}
$$

The stop criterion could be given (not the only conceivable one) by the following inequation:

$$
\frac{\sum_{t=1}^{T}\left(y_{t}-y_{t}^{k}\right)^{2}}{\sum_{t=1}^{T} y_{t}^{2}}<\varepsilon
$$

where $\varepsilon$ is small enough to satisfy the approximation precision needs.

The stop criterion is based on the idea that the

- distance between the

$\circ$ approximating values and the

- approximated ones

becomes after $\mathrm{k}$ iterations sufficiently (relatively) small as compared to the approximated values (supposing that the approximation method is convergent).

\section{The $\varphi$ function}

As defined in (4), $\varphi_{t} \stackrel{\text { def }}{=} \frac{a}{\left(b *\left(x-x_{t}\right)^{2}+1\right)}$; this function has a maximum value of $a_{t}$ in $x_{t}$ and tends to zero as $\mathrm{x}$ tends to $\pm \infty$. Depending on $b_{t}$, it's value becomes $\frac{a_{t}}{2}$ when $x=$ 
$\pm \frac{1}{\sqrt{b}}$ and becomes $\frac{a_{t}}{10}$ when $x= \pm \frac{3}{\sqrt{b}}$. For simplicity, $\mathrm{a}=1$ and $\mathrm{b}=1$ leads to the graphic presented in Figure 3.

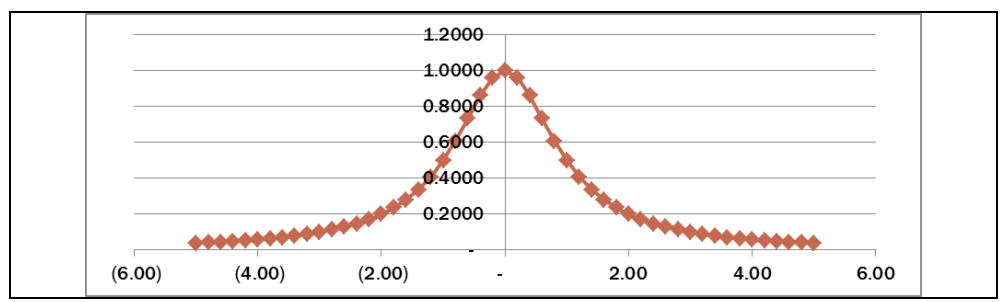

Figure 3: One-dimensional $\varphi$ function representation

\section{The convergence of the approximation to the approximated function}

As the approximated function is known only through its $\mathrm{T}$ values (approximation points and their corresponding values) the convergence could be discussed only within the limits of the convergence of the approximating function to the corresponding known values in the approximation points.

The degree of convergence is measured by the STOP criterion (15). At the moment of the submittance of this article, no formal demonstration of the convergence was available. However, beyond the intuitive convergence due to the fact that for each iteration the next approximation is made for the previous approximated difference, strong experimental data presented in the next chapter supports this hypothesis. One of the author's objectives is to further formally prove the convergence.

\section{Testing the proposed approximation method}

For simplicity reasons and without losing the generality, the testing was done for 1dimensional functions.

The to-be approximated values were generated using 52 types of functions, for which values were calculated in randomly generated points and with randomly generated coefficients for a total of $\mathbf{1 0 0 0}$ cases.

The Distance between the approximating function and the approximated one has been defined through the following function - see (15):

$$
D=\frac{\sum_{t=1}^{T}\left(y_{t}-y_{t}^{k}\right)^{2}}{\sum_{t=1}^{T} y_{t}^{2}}
$$

The smaller D (the approximation error), the higher the degree of convergence. D was calculated after 10 approximation iterations.

The Figure 4 displays the distribution of $\mathrm{D}$ after the 10 approximation iterations. 


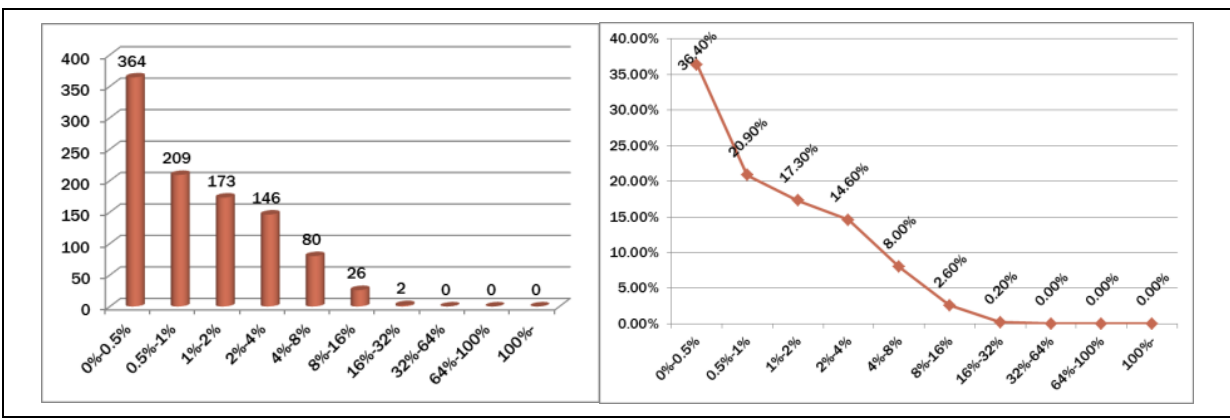

Figure 4: D (approximation error) distribution for 1000 randomly generated cases (left); the same, percentage representation (right)

This shows that after not more than 10 iterations, only:

- $2.8 \%$ of the cases had a lower convergence corresponding to D between $8 \%$ and $32 \%$.

- About $8 \%$ of the cases had a convergence corresponding to D between $4 \%$ and $8 \%$ and

- the rest of $89.2 \%$ of cases had a convergence corresponding to D between $0 \%$ and $4 \%$.

Table 1 shows the convergence depending on the type of function approximated.

Table 1: Approximation errors for the 52 types of functions after 10 iterations

\begin{tabular}{|c|c|c|c|}
\hline & Function Shape Code & Count & Approximation error $\mathrm{D}$ \\
\hline 1 & $-2,0,2,0,-1,1,0$ & 1 & $21.495 \%$ \\
\hline 2 & $2,-1,0,0,1,-2,0$ & 1 & $10.683 \%$ \\
\hline $3 \ldots 8$ & $\begin{array}{l}6 \text { different types of functions } \\
\text { representing } 60 \text { cases out of } 1000 \\
\text { have D between } 5 \% \text { and } 7 \%\end{array}$ & 2 & $5 \%-7 \%$ \\
\hline $9 \ldots 28$ & $\begin{array}{l}20 \text { different types of functions, } \\
\text { representing } 674 \text { cases of the } 1000\end{array}$ & 19 & $1 \%-5 \%$ \\
\hline $29 \ldots .52$ & $\begin{array}{l}24 \text { different types of functions } \\
\text { representing } 264 \text { cases of the } 1000\end{array}$ & 5 & $<1 \%$ \\
\hline
\end{tabular}

With the exception of the first two types of functions having the shape presented sketchlike in Figure 5 - which present a lower degree of convergence (a higher approximation error - $\mathrm{D}=21,495 \%$ and $\mathrm{D}=10,683$ ) - the average approximation error for the other types of graphics is below $8 \%$. 


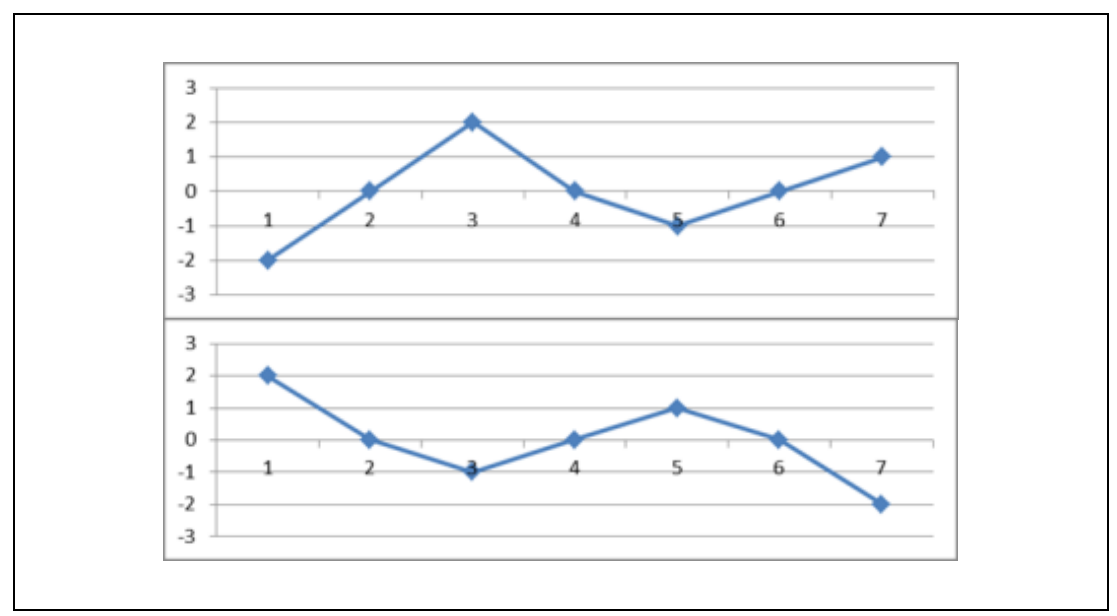

Figure 5: Lower convergence function shapes.

Figure 6 exhibits a number of examples where the approximated and the approximating functions (after 10 iterations) are represented together on the same graphic. Although the Function Shape Codes (FSC) are quite different, the approximations after 10 iterations (which use a maximum of 20 of 30 approximated points as input information) are quite good.

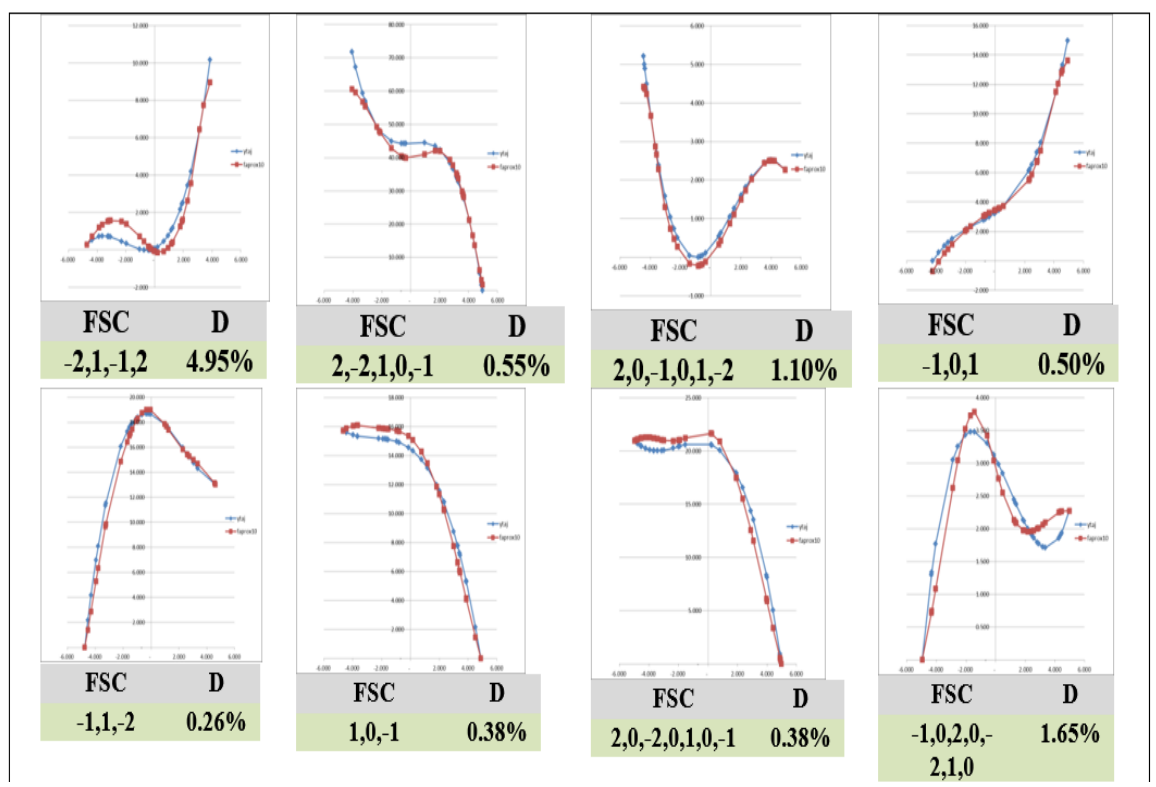

Figure 6: Eight examples of 1-dimensional function approximation 


\section{The Development of a Trainable Model $(* * *$, Wikipedia)}

A trainable model should be based on the gradual accumulation of as-much-as-possible non-redundant data, by using (2):

$$
\boldsymbol{x}_{\boldsymbol{i}+\mathbf{1}}-\boldsymbol{x}_{\boldsymbol{i}}=\boldsymbol{F}\left(\boldsymbol{x}_{\boldsymbol{i}}\right) *\left(\boldsymbol{t}_{\boldsymbol{i}+\mathbf{1}}-\boldsymbol{t}_{\boldsymbol{i}}\right)
$$

Each new input-state-output vector $\mathrm{x}$ (called reference vector) should be tested against the model's capability to predict the state based on the previous state.

If the prediction error is below an acceptable threshold, no learning based on this vector should take place.

If the model's capability to predict is affected by an error above the chosen threshold, the new vector should be learned and added to the existing set of reference vectors and the model (the function $\mathrm{F}$ - in fact each of its components $f_{i}$ of $\mathrm{F}$ ) should be re-approximated through the $\varphi$ functions associated to each reference vectors $\mathbf{x}$.

If associated to a vector $\mathrm{x}$ we find two different successor vectors $x_{s 1}$ and $x_{s 2}$, the interpretation would be that we cannot decide if the prediction should be $x_{s 1}$ or $x_{s 2}$.

In order to discriminate and choose correctly, additional information is needed and such information should take the form of a "hidden" parameter that would increase the dimension of the input-state-output space by one. Further system evolution should include the values of the new parameter. So inability to predict is a good clue that the system's model is based on an incomplete set of parameters.

\section{Applications in management. Further research}

From a management point of view, the main benefit of developing such a system based on the proposed approximation method resides in the possibility to create models, simulate and predict the evolution of the modeled systems, may it be the evolution of a product in a market, of a company in its environment or of another economic sub-system of interest.

Examples of candidate subjects for using the proposed modeling method could be:

(i) market reaction (adoption curve) when introducing a new product based on former experience for similar products (not disruptive ones)

(ii) a company, considering its input-status-output main indicators; possible results: short term predictions on evolution in case of input changes and no additional control loop

(iii) the subsystem formed by a node in a national economy (all the companies having the same activity code), in connection with its neighbouring nodes (suppliers, customers, authorities). The node would be described through a set of parameters such as: input vector from its supplier nodes, performance indicators, output vector for its customers, output to authorities. Possible results would include: short-term predictions regarding the evolution of the node when input vector values are changing.

(iv) the subsystem of currencies and main merchandises quoted by a national bank and corresponding exchanges and its evolution. 
The list is scarcely scratching the surface of possible applications.

The next research steps would include:

(i) Improving the existing model and increasing convergence speed

(ii) Evaluate the impact of using a continuous approximating model for maybe discontinuous or even discrete approximated realities

(iii) Formally demonstrating convergence

(iv) Extending experimental trials for $\mathrm{n}$-dimensional data

(v) Developing a trainable model for $n$-dimensional data that should allow the confrontation of real-life economic sub-systems and their models. Success in this case would bring the benefit of a certain degree of predictability.

\section{CONCLUSIONS}

The proposed approximation method provides good results after a small number of iterations and based on around only $2 / 3$ of the approximated points information (not all points' coordinates are used in the formulas - and of course this shows that some approximated points bring redundant information). So it seems to have a fast convergent approximating function that uses a reduced set of data (an efficient use of existing information).

Although no formal demonstration of convergence is available, there is strong experimental (randomly generated cases based) evidence that supports the intuitive hypothesis that the proposed approximation is convergent.

The model presented has appealing managerial implications by simulate and predict the evolution of the modeled systems: may it be the evolution of a product in a market, a company in its environment or another organization of interest.

\section{ACKNOWLEDGEMENT:}

The author would like to thank Professor Cezar Scarlat for his constant and inspired orientation and support for the development of this paper.

\section{REFERENCES}

Keilis-Borok, Vladimir ; Soloviev, Alexandre ; Lichtman, Allan (2011). Predictability of Extreme Events in Socio-economic and Political Complex Systems, Complex Systems in Finance and Econometric, p 273-289. Retrieved Sep 23rd 2014 from http://link.springer.com/referenceworkentry/10.1007\%2F978-0-387-30440-3_196 ; Springer New York

Wikipedia, the free encyclopedia (2004-2015). Economic model. Retrieved Sep 23rd 2014 from https://en.wikipedia.org/wiki/Economic_model ;

Wikipedia, the free encyclopedia(2002-2015). Dynamical system. Retrieved Sep 23rd 2015 from https://en.wikipedia.org/wiki/Dynamical_system ; 
Atkinson, Kendall; Han, Weimin; Stewart, David; (2009). Numerical Solution of Ordinary Differention Equations . Retrieved Sep 23rd 2015 from http://homepage.math.uiowa.edu/ atkinson/papers/NAODE_Book.pdf ; John Wiley \& Sons, Inc.

Judd, L. Keneth (2012). Approximation Methods. Retrieved Jul 17th 2012 from http://ice.uchicago.edu/2012_presentations/Faculty/Judd/Approximation_Methods_2012.p df ; Hoover Institution

Wikipedia, the free encyclopedia (2003-2015). Machine learning . Retrieved Jul 17th 2012 from https://en.wikipedia.org/wiki/Machine_learning; 